The Astrophysical Journal, 567:940-946, 2002 March 10

(C) 2002. The American Astronomical Society. All rights reserved. Printed in U.S.A.

\title{
DETECTION OF H I 21 CENTIMETER LINE ABSORPTION IN THE WARM NEUTRAL MEDIUM AND IN THE OUTER ARM OF THE GALAXY
}

\author{
K. S. DWARAKanath \\ Raman Research Institute, Bangalore 560 080, India; dwaraka@rri.res.in \\ AND \\ C. L. Carilli and W. M. Goss \\ National Radio Astronomy Observatory, P.O. Box O, Socorro, NM 87801; ccarilli@aoc.nrao.edu, mgoss@aoc.nrao.edu \\ Received 2001 September 3 ; accepted 2001 November 16
}

\begin{abstract}
Using the Westerbork Synthesis Radio Telescope, we have detected H I $21 \mathrm{~cm}$ line absorption in the warm neutral medium of the Galaxy toward the extragalactic source 3C 147. This absorption, at a local standard of rest (LSR) velocity of $-29 \pm 4 \mathrm{~km} \mathrm{~s}^{-1}$ with a full width at half-maximum of $53 \pm 6 \mathrm{~km} \mathrm{~s}^{-1}$, is associated with the Perseus arm of the Galaxy. The observed optical depth is $(1.9 \pm 0.2) \times 10^{-3}$. The estimated spin temperature of the gas is $3600 \pm 360 \mathrm{~K}$. The volume density is $0.4 \mathrm{~cm}^{-3}$ assuming pressure equilibrium. Toward two other sources, 3C 273 and 3C 295, no wide $\mathrm{H}$ I $21 \mathrm{~cm}$ line absorption was detected. The highest of the $3 \sigma$ lower limits on the spin temperature of the warm neutral medium is $2600 \mathrm{~K}$. In addition, we have also detected H I $21 \mathrm{~cm}$ line absorption from high-velocity clouds in the outer arm toward 3C 147 and 3C 380 at LSR velocities of $-117.3,-124.5$, and $-113.7 \mathrm{~km} \mathrm{~s}^{-1}$, respectively. We find two distinct temperature components in the high-velocity clouds with spin temperatures of greater than $1000 \mathrm{~K}$ and less than $200 \mathrm{~K}$, respectively.

Subject headings: Galaxy: disk - Galaxy: fundamental parameters - ISM: atoms - ISM: structure radio lines: ISM
\end{abstract}

\section{INTRODUCTION}

The early H I $21 \mathrm{~cm}$ line absorption and emission studies of the Galaxy led to the two-phase model of the interstellar medium in which dense cool $\mathrm{H}$ I clouds (the cold neutral medium) were in pressure equilibrium with a warm lowdensity medium (the warm neutral medium [WNM]) (Clark, Radhakrishnan, \& Wilson 1962; Clark 1965; Radhakrishnan et al. 1972). The mean spin temperature of the cold clouds was estimated to be $80 \mathrm{~K}$. Only a lower limit to the spin temperature of the WNM of $1000 \mathrm{~K}$ was obtained from an upper limit to the optical depth of $\mathrm{H}_{\mathrm{I}} 21 \mathrm{~cm}$ line absorption in the WNM. Since then there have been several attempts to measure the optical depth of the WNM (Mebold \& Hills 1975; Kalberla, Mebold, \& Reich 1980; Liszt, Dickey, \& Greisen 1982; Mebold et al. 1982; Payne, Salpeter, \& Terzian 1983; Kulkarni et al. 1985). Using the Bonn Telescope, Mebold \& Hills (1975) estimated an optical depth for the WNM based on the lack of $\mathrm{H}$ I emission in observations toward Cygnus A. They estimated the spin temperature of the WNM to be in the range of 3000$8000 \mathrm{~K}$ in different velocity ranges toward Cygnus A. No absorption in the WNM was detected in any of the other observations mentioned above, leading to upper limits on its optical depth and hence lower limits to the spin temperature. The highest of these lower limits to the spin temperature is $10^{4} \mathrm{~K}$ (Kulkarni et al. 1985).

Of all the phases of the interstellar medium, the WNM remains the least understood. Although $\mathrm{H}_{\mathrm{I}} 21 \mathrm{~cm}$ line emission from the WNM is detected easily in most directions, an accurate value for the temperature of the WNM is lacking. The spin temperature of the WNM is an important parameter with implications for models of the interstellar medium. The most detailed analysis of the equilibrium state of neutral hydrogen in the interstellar medium is the work of Wolfire et al. (1995). The primary heating mechanism is photoelectric emission from dust grains, while cooling is dominated by fine structure lines from heavy elements in colder regions and by hydrogen recombination lines in warmer regions. Recently, we detected H I $21 \mathrm{~cm}$ line absorption in the WNM using the Westerbork Synthesis Radio Telescope (WSRT). The absorption was detected toward Cygnus A at local standard of rest (LSR) velocities of -40 and $-70 \mathrm{~km} \mathrm{~s}^{-1}$ (Figs. 3 and 4 and Table 1 in Carilli, Dwarakanath, \& Goss 1998). These two velocity ranges were previously identified as being relatively free of cold absorbing clouds. The measured optical depth for the WNM along the line of sight to Cygnus $A$ is $(8.9 \pm 1.9) \times 10^{-4}$ at $-70 \mathrm{~km} \mathrm{~s}^{-1}$ and $(8.5 \pm 2.0) \times 10^{-4}$ at $-40 \mathrm{~km} \mathrm{~s}{ }^{-1}$, with corresponding spin temperatures of $6000 \pm 1700$ and $4800 \pm 1600 \mathrm{~K}$, respectively. The volume filling factor for the WNM appears to be fairly high and is $\sim 0.4$ (Carilli, Dwarakanath, \& Goss 1998). The measured value of the spin temperature toward Cygnus A is consistent with the range of kinetic temperature $(5500-8700 \mathrm{~K})$ for the WNM in the model proposed by Wolfire et al. (1995). While the current data toward Cygnus A are consistent with this picture, values of the spin temperature of the WNM in more directions are needed to provide a constrained physical model of the interstellar medium.

Mebold et al. (1981) have carried out a survey in Galactic $\mathrm{H}$ I absorption and emission toward a large number of extragalactic radio sources. From this list we have chosen four sources that are strong enough $(>10 \mathrm{Jy}$ at $20 \mathrm{~cm})$ to detect $\mathrm{H}$ I absorption (optical depth $\sim 0.001$ ) in an integration time of $\sim 12 \mathrm{hr}$. The $\mathrm{H}$ I emission profile toward each of these sources shows a few narrow lines plus a broad line component. The existing $\mathrm{H}$ I absorption spectrum toward each of these sources, however, has only narrow components corresponding to the narrow emission lines (Mebold et al. 1982). These absorption components (optical 
depth $>0.05)$ are due to the cold gas $\left(T_{s} \sim 80 \mathrm{~K}\right)$ in the Galaxy. The motivation of the present observations is to detect the $\mathrm{H}$ I absorption corresponding to the broad line in the $\mathrm{H} \mathrm{I}$ emission profile arising from the intercloud medium (warm neutral medium). The four sources chosen for the present observations are at $b>10^{\circ}$. These higher latitudes are relatively free of the cold neutral medium, and because of this, $\mathrm{H}$ I absorption measurements toward high-latitude sources are less confused by the plethora of narrow absorption lines routinely detected in the Galactic plane.

The observations are discussed in $\S 2$. The data analyses and the results obtained are given in $\S 3$. The implications for the determinations of the temperature and the density of the warm neutral medium based on the current observation are discussed in $\S 4.1$. A by-product of the current obser-

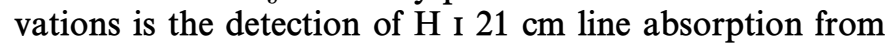
high-velocity clouds in the outer arm of the Galaxy. A discussion on this can be found in $\S 4.2$.

\section{OBSERVATIONS}

The $\mathrm{H}$ I $21 \mathrm{~cm}$ line absorption measurements were carried out toward 3C 147, 3C 273, 3C 295, and 3C 380 using the WSRT in 1998 and 2000. Some details of the observations are given in Table 1. The observations used a bandwidth of $1.25 \mathrm{MHz}$ centered at the frequency of the $\mathrm{H}$ I $21 \mathrm{~cm}$ line and 256 spectral channels. The resulting velocity resolution is $\sim 2.1 \mathrm{~km} \mathrm{~s}^{-1}$ after Hanning smoothing. Absolute flux density calibration was obtained by observing 3C 286 and 3C 48. Since the sources of interest were bright and unresolved at the WSRT resolutions $\left(\sim 15^{\prime \prime}\right)$, amplitude and phase calibrations of the antennas were carried out through self-calibration. The bandpass calibration was obtained through frequency switching on the source itself. For this purpose, each "on-line" scan of 25 minutes duration was sandwiched between two "off-line" scans each of 25 minutes duration. These off-line scans were offset in frequency from the on-line scan by $\pm 1.25 \mathrm{MHz}$, respectively. The off-line scans are unaffected by contamination due to Galactic $\mathrm{H}$ I and provide an accurate bandpass.

The data were analyzed using the Astronomical Image Processing System (AIPS) developed by the National Radio Astronomy Observatory. For each source, a continuum database was constructed by averaging the line-free channels. The amplitude and phase solutions of antennas obtained through normal and self-calibration of this data set were transferred to the spectral line data. The calibrated and bandpass corrected spectral line data of the source were used in further analysis. The continuum flux density in the source visibilities was removed by making a linear fit to the visibilities in the line-free channels as a function of channel and subtracting it from the source visibilities in all the spectral channels. These continuum-subtracted visibilities were used to make spectral cubes of $\mathrm{H}$ I absorption images. The spectral cubes were CLEANed. The rms per channel was typically $\sim 5 \mathrm{mJy}^{\text {beam }}{ }^{-1}$. In order to avoid any possible contamination due to Galactic $\mathrm{H}$ I emission, visibilities in the short spacings (the inner $\sim 0.5 \mathrm{~km}$ ) were not used in making the $\mathrm{H}$ I absorption images.

Since the aim of the current observations is to detect weak (optical depth $\sim 10^{-3}$ ) and wide (width $\sim 50 \mathrm{~km} \mathrm{~s}^{-1}$ ) lines, the most important requirement is the stability of the bandpass. This stability can be estimated by comparing the two off-line bandpasses from the same source in a given observing run. Such a comparison for 3C 147, 3C 273, and 3C 295 indicated that the two off-line bandpasses on a given source are consistent with each other to within $2 \times 10^{-4}$ $(1 \sigma)$. The calibrated on-line data of these sources is not expected to have any spurious features beyond this level. This stability is greater than or comparable to the rms noise in the optical depth images toward these three sources (Table 1). In the case of 3C 380, the two off-line bandpasses differed at the level of $6 \times 10^{-4}(1 \sigma)$. In addition, in the case of 3C 380, many antennas showed variations across the passband with periods of $\sim 0.5 \mathrm{MHz}\left(\sim 100 \mathrm{~km} \mathrm{~s}^{-1}\right)$. Due to the limited dynamic range achieved, the 3C 380 data were not considered for wide-line analysis. However, these data were suitable for an investigation of narrow $\mathrm{H} \mathrm{I}$ absorption lines.

\section{RESULTS}

The $\mathrm{H}$ I absorption spectra toward the four sources in Table 1 are displayed in the lower panels in Figures 1-4, respectively. The corresponding $\mathrm{H}$ I emission profiles from the Leiden Dwingeloo Sky Survey (LDSS) (Hartmann \& Burton 1997) are in the upper panels of these figures. A Gaussian decomposition of these profiles was carried out using the Groningen Image Processing System (GIPSY). Each optical depth spectrum was Hanning smoothed and simultaneously fit with the minimum number of Gaussians until the reduced $\chi^{2}$ value was $\leq 1$. In each case, the model spectrum was then subtracted from the observed spectrum to obtain the residual spectrum. The best-fit model spectrum shows a featureless residual spectrum. The residual spectra so obtained toward the sources $3 \mathrm{C} 147,3 \mathrm{C} 273$, and 3C 295 are shown in Figure 7. Similar analyses were also carried out on the corresponding $\mathrm{H}$ I emission spectra. The parameters of the best-fit Gaussians from these analyses are summarized in Table 2. In many cases, the central velocities of $\mathrm{H} \mathrm{I}_{\mathrm{I}}$ absorption and $\mathrm{H} \mathrm{I}$ emission components agree within errors. In such cases, we assume that the absorption

TABLE 1

WSRT OBSERVATIONS

\begin{tabular}{ccccccc}
\hline \hline Source & $\begin{array}{c}\text { Galactic Coord. } \\
(\mathrm{deg})\end{array}$ & $\begin{array}{c}S_{1420} \\
(\mathrm{Jy})\end{array}$ & $\begin{array}{c}\text { Synthesized Beam FWHM } \\
(\mathrm{arcsec})\end{array}$ & $\begin{array}{c}\text { P.A. } \\
(\mathrm{deg})\end{array}$ & $\begin{array}{c}\text { rms } \\
(\mathrm{mJy} \text { beam }\end{array}$
\end{tabular}

NoтE.-All the observations had a velocity resolution of $2.1 \mathrm{~km} \mathrm{~s}^{-1}$ and 128 channels after Hanning smoothing. The rms quoted is per channel. The last column indicates the approximate total time spent on each observation. The observations were carried out during 1998 December, 2000 March, and 2000 May. 

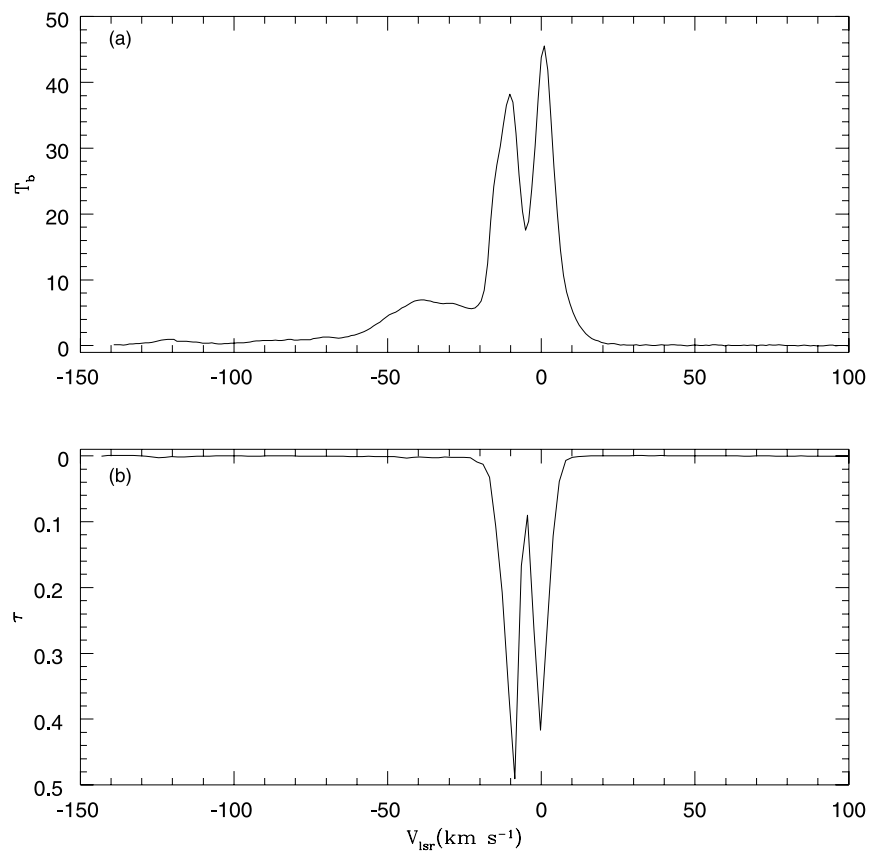

FIG. 1.-(a) $\mathrm{H} \mathrm{I}_{\mathrm{I}}$ emission at $l=161^{\circ} .5, b=10^{\circ} .5$ from the LDSS (Hartmann \& Burton 1997). The LDSS has a resolution of $\sim 35^{\prime}$, an rms of $\sim 0.07 \mathrm{~K}$, and a velocity resolution of $\sim 1 \mathrm{~km} \mathrm{~s}^{-1}$. (b) $\mathrm{H}$ I absorption toward 3C 147 from the current observations using the Westerbork Synthesis Radio Telescope (WSRT). The WSRT observations have a synthesized beam of $16^{\prime \prime} .6 \times 12^{\prime \prime} .1$ (P.A. $=-2.4$ ), an rms of $1.5 \times 10^{-4}$ in $\tau$, and a velocity resolution of $\sim 2.1 \mathrm{~km} \mathrm{~s}^{-1}$. The $x$-axis is velocity in the local standard of rest.

and emission arise from the same $\mathrm{H}$ I. An estimate of the column density, the spin temperature, and the width expected due to thermal broadening is also given for these components. If the central velocities do not agree within the
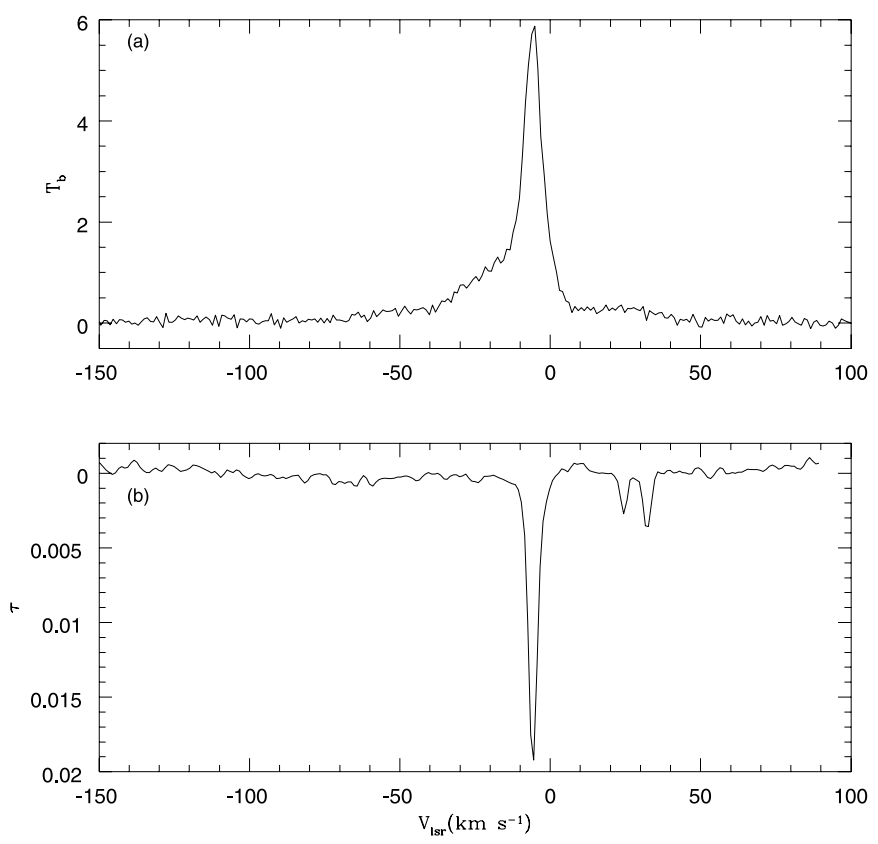

FIG. 2.-(a) $\mathrm{H}$ I emission at $l=290.0, b=64.5$ from LDSS. (b) $\mathrm{H}$ I absorption toward 3C 273 from the WSRT. The WSRT observations have a synthesized beam of $511^{\prime \prime} \times 11^{\prime \prime} 7($ P.A. $=0.4)$, an rms of $1.2 \times 10^{-4}$ in $\tau$, and a velocity resolution of $\sim 2.1 \mathrm{~km} \mathrm{~s}^{-1}$.
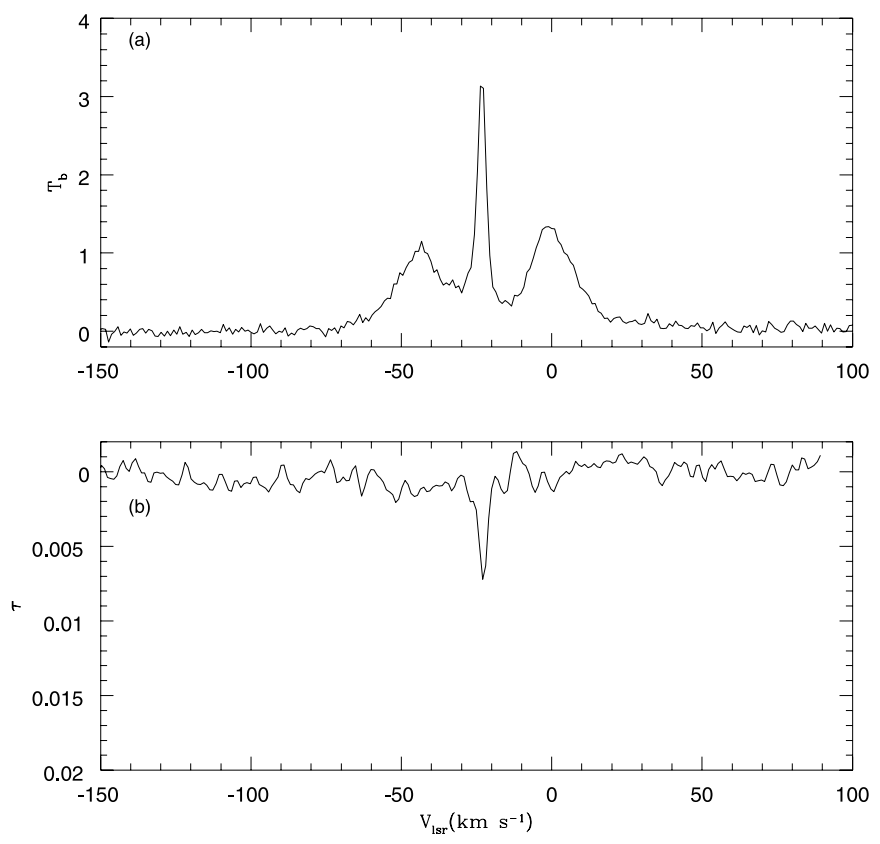

FIG. 3. - (a) $\mathrm{H}$ I emission at $l=97.5, b=61.0$ from LDSS. (b) $\mathrm{H} \mathrm{I}$ absorption toward 3C 295 from the WSRT. The WSRT observations have a synthesized beam of $28^{\prime \prime} .9 \times 14^{\prime \prime} 4$ (P.A. $=-75^{\circ} .0$ ), an $\mathrm{rms}$ of $3.6 \times 10^{-4}$ in $\tau$, and a velocity resolution of $\sim 2.1 \mathrm{~km} \mathrm{~s}^{-1}$.

quoted errors, either the absorption or the emission column is left blank, indicating that they arise from different $\mathrm{H} \mathrm{I}$.

In Figures 5 and 6, the $\mathrm{H}$ I absorption spectra toward 3C 147 and 3C 380 are shown along with the corresponding $\mathrm{H} \mathrm{I}$ emission spectra, respectively. Toward the direction of $3 \mathrm{C}$ 147 , note the weak H I absorption (Fig. $5 b$ ) over the velocity range $-70 \mathrm{~km} \mathrm{~s}^{-1}<V_{1 \mathrm{sr}}<-30 \mathrm{~km} \mathrm{~s}^{-1}$ and a corresponding $\mathrm{H}_{\mathrm{I}}$ emission (Fig. 5a). The weak $\mathrm{H}_{\mathrm{I}}$ absorption detected toward 3C 147 over the velocity range
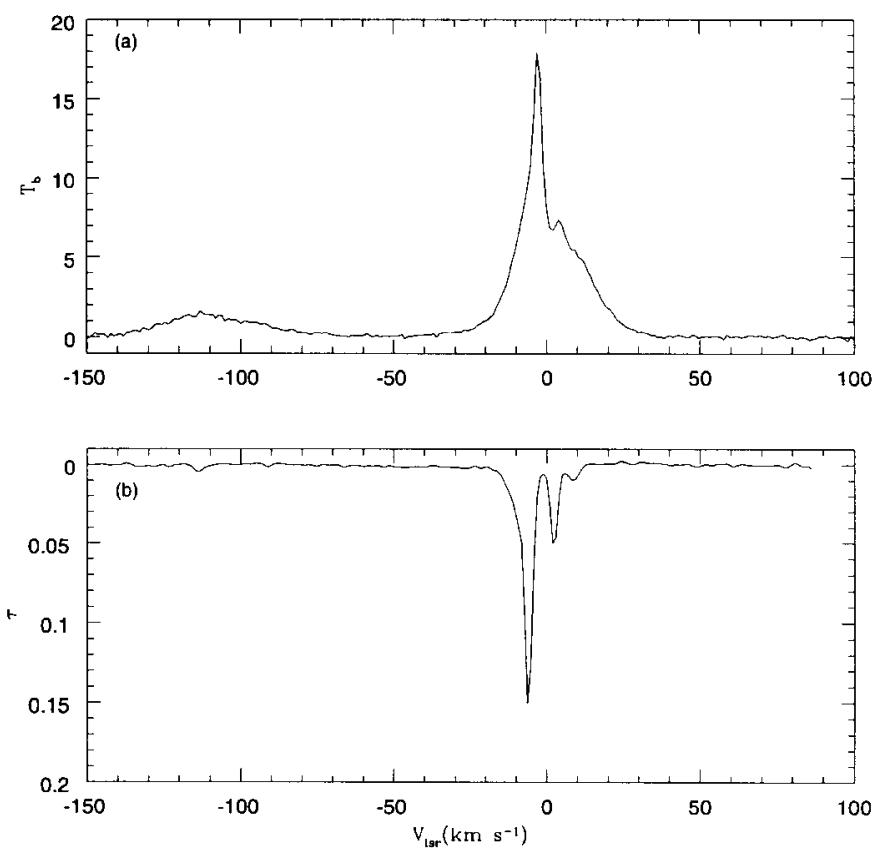

FIG. 4.-(a) $\mathrm{H} \mathrm{I}$ emission at $l=77^{\circ} 0, b=23.5$ from LDSS. (b) $\mathrm{H}_{\mathrm{I}}$ absorption toward 3C 380 from the WSRT. The WSRT observations have a synthesized beam of $46^{\prime \prime} 1 \times 19^{\prime \prime} .7$ (P.A. $=16^{\circ} .1$ ), an rms of $5.2 \times 10^{-4}$ in $\tau$, and a velocity resolution of $\sim 2.1 \mathrm{~km} \mathrm{~s}^{-1}$. 
TABLE 2

BeST-FIT Gaussian COMPONENTS

\begin{tabular}{|c|c|c|c|c|c|c|c|c|c|}
\hline \multirow[b]{2}{*}{ SOURCE } & \multicolumn{3}{|c|}{ H I ABSORPTION } & \multicolumn{4}{|c|}{ H I EMISSION } & \multirow[b]{2}{*}{$\begin{array}{c}T_{s} \\
(\mathrm{~K})\end{array}$} & \multirow[b]{2}{*}{$\begin{array}{c}\delta V_{D} \\
\left(\mathrm{~km} \mathrm{~s}^{-1}\right)\end{array}$} \\
\hline & $\begin{array}{c}V_{1 \mathrm{sr}} \\
\left(\mathrm{km} \mathrm{s}^{-1}\right)\end{array}$ & $\begin{array}{c}\delta V_{1 / 2} \\
\left(\mathrm{~km} \mathrm{~s}^{-1}\right)\end{array}$ & $\tau^{\mathrm{a}}$ & $\begin{array}{c}V_{1 \mathrm{sr}} \\
\left(\mathrm{km} \mathrm{s}^{-1}\right)\end{array}$ & $\begin{array}{c}\delta V_{1 / 2} \\
\left(\mathrm{~km} \mathrm{~s}^{-1}\right)\end{array}$ & $\begin{array}{c}T_{b} \\
(\mathrm{~K})\end{array}$ & $N_{\mathrm{H}_{\text {I }}}$ & & \\
\hline \multirow[t]{12}{*}{$3 \mathrm{C} 147 \ldots \ldots$} & $+2.8(.5)$ & $5.0(.4)$ & $.1(19)$ & $+5.6(2)$ & $11.1(2)$ & $8.5(2)$ & 1.8 & 85 & 2.0 \\
\hline & $-0.6(.1)$ & $4.6(.1)$ & $.38(21)$ & $+0.7(.04)$ & $6.5(.2)$ & $39.5(4.1)$ & 5.0 & 105 & 2.2 \\
\hline & $-8.8(.01)$ & 2.9(.04) & $.29(5)$ & & & & & $<185$ & \\
\hline & $-10.7(.06)$ & 7.3(.06) & $.26(3)$ & $-10.8(.04)$ & $9.6(.1)$ & $35.9(.2)$ & 6.7 & 140 & 2.5 \\
\hline & $-20.2(.07)$ & $2.4(.8)$ & $.009(.7)$ & & & & & $<125$ & \\
\hline & $-29(4)$ & $53(6)$ & $.0019(.2)$ & $-35.5(.3)$ & $34(1.1)$ & $6.9(.1)$ & 4.6 & 3600 & 12.8 \\
\hline & $-34.7(.8)$ & $2.7(3.9)$ & $.0013(1.5)$ & & & & & $<160$ & \\
\hline & $-43.9(.6)$ & $3.5(1.2)$ & $.0016(.5)$ & & & & & $<270$ & \\
\hline & & & & $-81.8(2.2)$ & $29(6)$ & $0.8(.1)$ & 0.5 & $>1780$ & \\
\hline & & & & $-120.4(1.4)$ & $17(3.6)$ & $0.8(.1)$ & 0.3 & $>1780$ & \\
\hline & $-117.3(.3)$ & $8.0(.9)$ & $.0013(.1)$ & & & & & $<1400$ & \\
\hline & $-124.5(.1)$ & $3.5(.2)$ & $.003(.1)$ & & & & & $<270$ & \\
\hline \multirow[t]{6}{*}{$3 C 273 \ldots \ldots$} & $+32.2(.08)$ & $3.0(.2)$ & $.0038(.2)$ & & & & & $<200$ & \\
\hline & $+24.5(.1)$ & $2.6(.3)$ & $.0026(.2)$ & & & & & $<150$ & \\
\hline & $-5.4(.1)$ & $6.6(.6)$ & $.0048(1.1)$ & & & & & $<950$ & \\
\hline & $-5.8(.03)$ & $3.0(.1)$ & $.0147(1.1)$ & $-5.7(.03)$ & $6.8(.1)$ & $4.6(.06)$ & 0.6 & 300 & 3.7 \\
\hline & & & & $-12.7(1.6)$ & $76.0(8)$ & $0.34(.05)$ & 0.5 & $>900$ & \\
\hline & & & & $-13.2(.5)$ & 24.7(1.4) & $0.95(.06)$ & 0.5 & $>2600$ & \\
\hline \multirow[t]{3}{*}{$3 C 295 \ldots \ldots$} & & & & $-0.25(.2)$ & 19.1(.4) & $1.2(.02)$ & 0.4 & $>1100$ & \\
\hline & $-22.9(.1)$ & $3.5(.3)$ & $.0066(.5)$ & $-23.3(.03)$ & $3.9(.08)$ & 2.9(.05) & 0.2 & 440 & 4.5 \\
\hline & & & & $-41.9(.3)$ & $24.9(.7)$ & $0.9(.02)$ & 0.4 & $>800$ & \\
\hline \multirow[t]{8}{*}{$3 C 380 \ldots \ldots$} & $+8.4(.1)$ & $4.2(.3)$ & $.0098(.7)$ & & & & & $<385$ & \\
\hline & $+2.3(.02)$ & $2.8(.05)$ & $.0526(.8)$ & & & & & $<170$ & \\
\hline & & & & $+0.1(.1)$ & $26.6(.2)$ & $7.6(.1)$ & 3.9 & $>4200$ & \\
\hline & & & & $-3.1(.02)$ & $3.5(.07)$ & $10.1(.2)$ & 0.7 & $<270$ & \\
\hline & $-6.0(.01)$ & $2.7(.03)$ & $.1208(1.2)$ & & & & & $<160$ & \\
\hline & $-8.3(.1)$ & $8.3(.1)$ & $.0356(.1)$ & & & & & $<1510$ & \\
\hline & & & & $-109.7(.6)$ & 38.1(1.4) & $1.3(.04)$ & 1.0 & $>800$ & \\
\hline & $-113.7(.3)$ & $3.1(.6)$ & $.0043(.8)$ & & & & & $<210$ & \\
\hline
\end{tabular}

NoTE.-The H I emission components are best-fit to data from LDSS (Hartmann \& Burton 1997). The H I absorption data are from the current observations. $N_{\mathrm{HI}}$ is in units of $10^{20} \mathrm{~cm}^{-2} . \delta V_{1 / 2}$ is full width at half-maximum. The lower limits on $T_{s}$ are $3 \sigma$ limits based on the upper limits on $\tau$. The upper limits on $T_{s}$ correspond to the values if the line widths are entirely due to thermal broadening. In those cases where absorption and emission were detected from the same gas, the width due to pure thermal broadening is given by $\delta V_{D}$. The numbers in parentheses are $1 \sigma$ formal errors from the fitting procedure.

${ }^{\text {a }}$ The $1 \sigma$ errors obtained from fitting are given in parentheses in units of $10^{-3}$.

$-70 \mathrm{~km} \mathrm{~s}^{-1}<V_{\mathrm{lsr}}<-30 \mathrm{~km} \mathrm{~s}^{-1}$ (Fig. $5 b$ ) has a $10 \sigma$ significance compared to the bandpass stability observed in the 3C 147 observations. This $\mathrm{H}$ I absorption can be combined with the corresponding $\mathrm{H}$ I emission (Fig. 5a) to estimate the spin temperature of the $\mathrm{H}$ I gas responsible for them. The $\mathrm{H}$ I emission spectrum in $T_{b}$ toward 3C 147 (Fig. $5 a$ ) is divided by the optical depth values (Fig. $5 b$ ) to obtain spin temperature as a function of $V_{\mathrm{lsr}}$. Over the velocity range $-70 \mathrm{~km} \mathrm{~s}^{-1}<V_{\mathrm{lsr}}<-30 \mathrm{~km} \mathrm{~s}^{-1}$ where the weak absorption is detected, the spin temperature has an average value of $3000 \pm 500 \mathrm{~K}$ ( $1 \sigma$ error $)$. The high spin temperature and the wide range of velocities over which $\mathrm{H} \mathrm{I}$ absorption is detected indicates that this $\mathrm{H}$ I gas is due to the warm neutral medium of the Galaxy. Note that the $\mathrm{H} \mathrm{I}$ absorption toward 3C 380 (Fig. 6b) also shows a similar "dip" over the velocity range $-50 \mathrm{~km} \mathrm{~s}^{-1}<V_{\mathrm{lsr}}<-20$ $\mathrm{km} \mathrm{s}^{-1}$. This feature has less than a $3 \sigma$ significance compared to the bandpass stability in the 3C 380 observations.

In Figure 7, the residual $\mathrm{H}$ i absorption spectra obtained after subtracting the best-fit models (Table 2) from the observed spectra toward 3C 147, 3C 273, and 3C 295 are shown in top to bottom panels, respectively. In the case of 3C 147 (Fig. 7a), the velocity range over which the simultaneous fit was carried out was restricted to $-110 \mathrm{~km} \mathrm{~s}^{-1}$
$<V_{\text {lsr }}<+100 \mathrm{~km} \mathrm{~s}^{-1}$ in order not to exceed the maximum number of Gaussians that GIPSY can fit simultaneously. The spectrum shown for 3C 147 (Fig. 7a) is restricted to this velocity range. A separate simultaneous fit was carried out with two Gaussians over the velocity range $-140 \mathrm{~km} \mathrm{~s}^{-1}<V_{1 \mathrm{sr}}<-90 \mathrm{~km} \mathrm{~s}^{-1}$ to account for the two high-velocity narrow absorption lines detected toward $3 \mathrm{C}$ 147 (Fig. $5 b$ and Table 2). There is a clear separation between these two velocity ranges of the spectrum with no spectral features in the overlapping region $-110 \mathrm{~km} \mathrm{~s}^{-1}<V_{1 \mathrm{sr}}<-90 \mathrm{~km} \mathrm{~s}^{-1}$ (Fig. 5b) enabling independent Gaussian analyses in the two velocity ranges. In Figure $7 a$ two spectra corresponding to 3C 147 are shown. The solid line indicates the residual absorption spectrum obtained after subtracting from the observed spectrum the model containing only the seven narrow $\mathrm{H}$ I absorption lines in the velocity range $-110 \mathrm{~km} \mathrm{~s}^{-1}<V_{1 \mathrm{sr}}<+100$ $\mathrm{km} \mathrm{s}^{-1}$ (Table 2). The broken line indicates the residual spectrum after including the wide $\mathrm{H}$ I absorption line $\left(V_{1 \mathrm{sr}}=-29 \pm 4 \mathrm{~km} \mathrm{~s}^{-1}\right.$ and $\left.\delta V_{1 / 2}=53 \pm 6 \mathrm{~km} \mathrm{~s}^{-1}\right)$ in addition to the seven narrow lines in the model spectrum. The reduced $\chi^{2}$ values of the residual spectra are 16 (solid line) and 0.6 (broken line), respectively. These two residual spectra and the corresponding reduced $\chi^{2}$ values clearly 

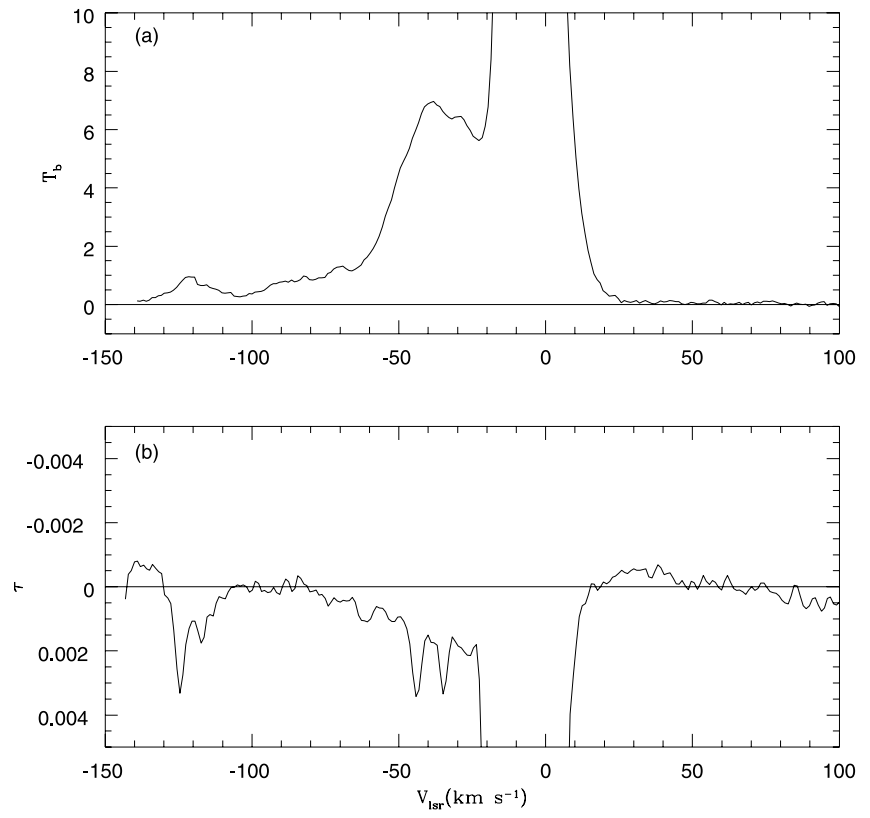

FIG. 5.-Same as Fig. 1 in an expanded $y$-scale. Note the weak $\mathrm{H}$ I absorption over the velocity range $-70 \mathrm{~km} \mathrm{~s}^{-1}<V_{1 \mathrm{sr}}<-30 \mathrm{~km} \mathrm{~s}^{-1}$ in (b) and a corresponding $\mathrm{H}$ I emission in (a). The implied spin temperature $\left(T_{b} / \tau\right)$ over this velocity range is $3000 \pm 500 \mathrm{~K}$.

indicate the existence of a wide $\mathrm{H}$ I absorption line toward $3 C$ 147. In addition, a corresponding wide $\left(\delta V_{1 / 2}=34\right.$ $\pm 1.1 \mathrm{~km} \mathrm{~s}^{-1}$ ) $\mathrm{H} \mathrm{I}$ emission component at a velocity $V_{\mathrm{lsr}}=$ $-35.5 \pm 0.3 \mathrm{~km} \mathrm{~s}^{-1}$ is observed in the LDSS (Fig. $5 a$ and Table 2). The central velocities of these absorption and emission lines agree to within $\sim 1 \sigma$. However, their widths are discrepant at the $3 \sigma$ level. We identify these two emission and absorption lines as arising from the same $\mathrm{H}$ I gas. The estimated spin temperature of this component is $3600 \pm 360 \mathrm{~K}$, in agreement with the direct estimate of spin temperature made in the previous paragraph by dividing
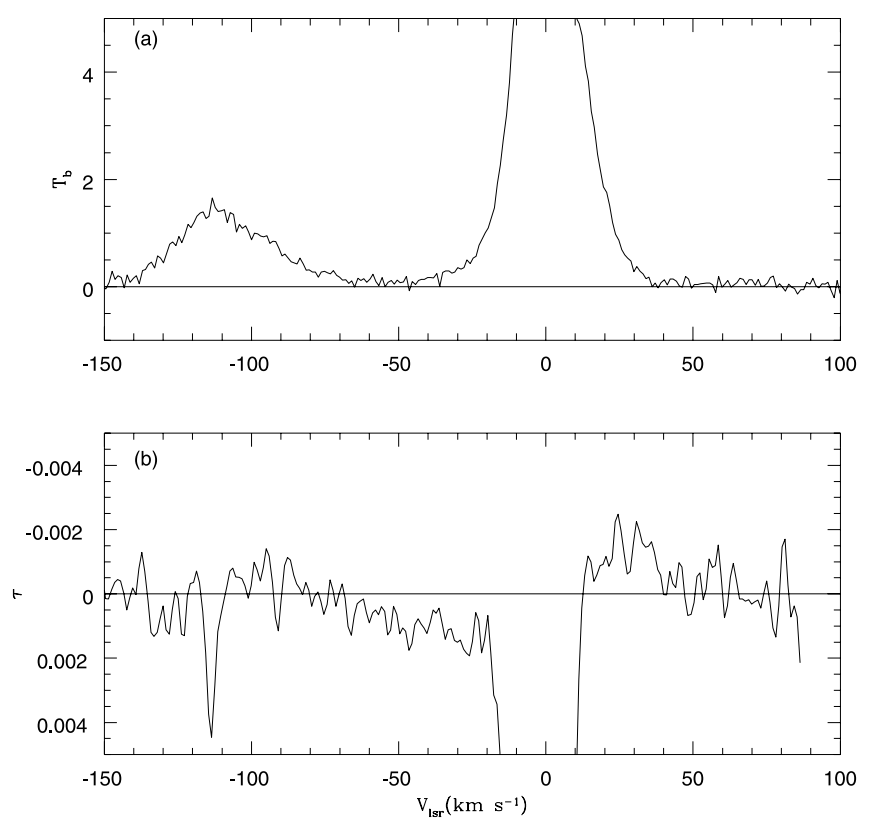

Fig. 6.-Same as Fig. 4 in an expanded $y$-scale
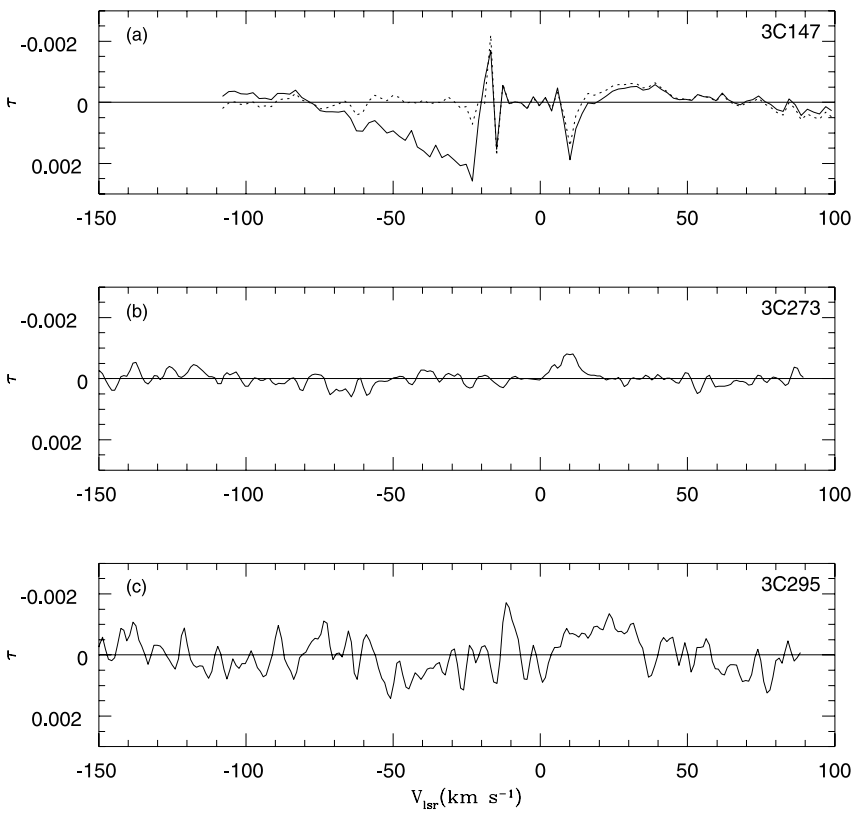

FIG. 7.- Residual $\mathrm{H}$ I absorption spectra (data-model) toward 3C 147 , 3C 273, and 3C 295. The parameters of the best-fit $\mathrm{H}$ I absorption Gaussian components used in the models are listed in Table 2. (a) The solid line shows the residual spectrum toward 3C 147 when the model included only the seven narrow $H$ I absorption lines in the velocity range $-110 \mathrm{~km} \mathrm{~s}^{-1}<V_{\text {lsr }}<100 \mathrm{~km} \mathrm{~s}^{-1}$. The broken line is the residual spectrum when the model included the wide absorption line of width $53 \mathrm{~km} \mathrm{~s}^{-1}$ at $V_{\mathrm{lsr}}=-29 \mathrm{~km} \mathrm{~s}^{-1}$. The sharp features at $V_{\mathrm{lsr}} \sim-15$ and $+10 \mathrm{~km} \mathrm{~s}^{-1}$ are artifacts left over from fitting multiple Gaussians to deep and narrow lines. (b) The residual spectrum toward 3C 273. The amplitudes of the artifacts in $(a)$ are a small percent of the peak optical depth and would not be detected in this spectrum. (c) The residual spectrum toward 3C 295.

the $\mathrm{H}$ I emission brightness temperature by the corresponding optical depth as a function of $V_{\mathrm{lsr}}$.

The residual spectra shown in Figures $7 b$ and $7 c$ corresponding to the $\mathrm{H}$ I absorption toward 3C 273 and 3C 295 do not indicate wide $\mathrm{H}$ I absorption, although the $\mathrm{H}$ I emission spectra toward these sources do show wide lines (Table 2 ). In these two cases upper limits to the optical depths of wide absorption lines can be obtained. Based on these upper limits and the $\mathrm{H}$ I column densities of the corresponding emission lines, lower limits to the spin temperatures of these wide emission lines are estimated (Table 2). For example, toward $3 \mathrm{C} 273$, a lower limit $(3 \sigma)$ of $2600 \mathrm{~K}$ is obtained for the emission line at $V_{\text {lsr }}=-13.2 \mathrm{~km} \mathrm{~s}^{-1}$ with a width of $\delta V_{1 / 2}=24.7 \pm 1.4 \mathrm{~km} \mathrm{~s}^{-1}$.

The H I absorption profile toward 3C 147 (Fig. 5b) also shows two narrow, weak $\mathrm{H}$ I absorption lines at $V_{\mathrm{lsr}}=$ -117.3 and $-124.5 \mathrm{~km} \mathrm{~s}^{-1}$ due to $\mathrm{H} \mathrm{I}$ in the outer arm of the Galaxy. The H I emission profile toward 3C 147 (Fig. $5 a$ ) has a line at $V_{\text {lsr }}=-120.4 \mathrm{~km} \mathrm{~s}^{-1}$ with a full width at half-maximum of $17 \mathrm{~km} \mathrm{~s}^{-1}$ (Table 2). It appears that this $\mathrm{H}$ I emission and the two $\mathrm{H}$ I absorption lines may be associated. However, since there is no exact correspondence of the central velocities and the widths of the emission and absorption lines, we can only estimate limits on the spin temperatures. Assuming that the widths of these two absorption lines are due to thermal broadening alone, upper limits to their spin temperatures of 1400 and $270 \mathrm{~K}$ are obtained, respectively. The absence of $\mathrm{H}$ I absorption corresponding to the $\mathrm{H} \mathrm{I}$ emission at $V_{\mathrm{lsr}}=-120.4 \mathrm{~km} \mathrm{~s}^{-1}$ leads to a lower limit of $1780 \mathrm{~K}$ to its spin temperature. 
Similarly, the H I absorption spectrum toward 3C 380 (Fig. $6 b$ ) has a discrete narrow line at $V_{\text {lsr }}=-113.7 \mathrm{~km} \mathrm{~s}^{-1}$ with an $\mathrm{H}$ I emission line in the LDSS spectrum at $V_{1 \mathrm{sr}}=$ $-109.7 \mathrm{~km} \mathrm{~s}^{-1}$ (Fig. 6a). However, H I emission line width is more than 10 times the $\mathrm{H}$ I absorption-line width (Table 2). The lower limit to the spin temperature of this wide emission line with no corresponding $\mathrm{H}$ I absorption is 800 $\mathrm{K}$. An upper limit to the spin temperature of $210 \mathrm{~K}$ can be estimated for the $\mathrm{H}$ I absorption at $V_{\mathrm{lsr}}=-113.7 \mathrm{~km} \mathrm{~s}^{-1}$, if the width is due entirely to thermal broadening.

\section{DISCUSSION}

\subsection{Temperature and Density}

The spin temperature of the $\mathrm{H}$ I gas is influenced by the intensity of radiation at the frequency of the $21 \mathrm{~cm}$ line; collisions with electrons, protons, and hydrogen atoms; and the intensity of the Ly $\alpha$ radiation field (Field 1958; Liszt 2001). In the context of the warm neutral medium, the most important factor influencing the spin temperature of the $\mathrm{H} \mathrm{I}$ gas is the $\mathrm{Ly} \alpha$ radiation field. The $\mathrm{H}$ I spin temperature is expected to be close to the kinetic temperature of the gas through the process of resonant scattering of ambient Ly $\alpha$ photons (Field 1958). The efficacy of this process depends on the ambient density of Ly $\alpha$ photons. There are no secure measurements of the diffuse Galactic Ly $\alpha$ photon density; an upper limit of $2.5 \times 10^{-6} \mathrm{~cm}^{-3}$ in the solar neighborhood has been estimated (Holberg 1986). The areal production rate of ionizing photons from early-type stars in the solar neighborhood is estimated to be $3.7 \times 10^{7}$ photons $\mathrm{cm}^{-2} \mathrm{~s}^{-1}$ (Vacca, Garmany, \& Shull 1996). Assuming this to be comparable to the production rate of $\operatorname{Ly} \alpha$ photons leads to a Galactic Ly $\alpha$ flux from early-type stars. Liszt (2001) discusses the relation between the spin temperature and the kinetic temperature as a function of the number density of H-nuclei, the gas pressure of the interstellar medium, and the fraction of Galactic Ly $\alpha$ flux from earlytype stars that permeates the diffuse gas.

If the fraction of $L y \alpha$ flux from early-type stars that permeates the warm gas is less than 0.0001 , the spin temperature is significantly lower than the kinetic temperature of the $\mathrm{H}$ I gas. The spin temperature of $3600 \mathrm{~K}$ estimated in the current observations implies a kinetic temperature of $7000 \mathrm{~K}$ and a density of $0.4 \mathrm{~cm}^{-3}$ in the two-phase model proposed by Wolfire et al. (1995) (Fig. 5 in Liszt 2001). On the other hand, if the fraction of Ly $\alpha$ flux from early-type stars that permeates the warm gas is large (greater than 0.01 ), the spin temperature is within $10 \%$ of the kinetic temperature. However, $\mathrm{H}$ I gas at such a low kinetic temperature as $3600 \mathrm{~K}$ does not have a stable phase and is outside the allowed range of values in the model proposed by Wolfire et al. (1995) (Fig. 5 in Liszt 2001). Either we are observing an unstable phase of $\mathrm{H} \mathrm{I}$, or very small, if any, fraction of $L y \alpha$ radiation from early-type stars permeates the diffuse intercloud medium.

The warm $\mathrm{H}_{\mathrm{I}}$ gas at $V_{\mathrm{lsr}} \sim-30 \mathrm{~km} \mathrm{~s}^{-1}$ is associated with the Perseus arm of the Galaxy at a distance of $2.5 \mathrm{kpc}$ $\left(l \sim 160^{\circ}, b \sim 10^{\circ}\right)$. The mean $z$ of the $\mathrm{H}$ I gas is thus $\sim 460$ pc. At this height the electron density is less than $0.02 \mathrm{~cm}^{-3}$ (Taylor \& Cordes 1993) and combined with the current estimate of $n_{\mathrm{H}}$ implies that the ionization fraction at this height is less than 0.05 . As discussed earlier in this section, the kinetic temperature of the WNM in the direction of $3 \mathrm{C}$ 147 can be in the range $3600-7000 \mathrm{~K}$. The full width at

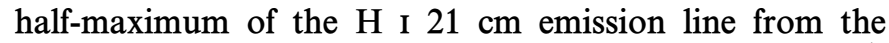
WNM due to thermal broadening alone is $13-18 \mathrm{~km} \mathrm{~s}^{-1}$. The observed $\mathrm{H}$ I emission line width from the WNM in the direction of $3 \mathrm{C} 147$ is $34 \mathrm{~km} \mathrm{~s}^{-1}$. If the observed excess width is attributed to turbulence in the WNM, the implied full width at half-maximum of the turbulence broadening is $31-29 \mathrm{~km} \mathrm{~s}^{-1}$.

\subsection{The Outer Arm and High-Velocity $\mathrm{H}$ I Gas}

The outer arm is the structure observed in $\mathrm{H} \mathrm{I}$ in the range $50^{\circ}<l<195^{\circ},-5^{\circ}<b<35^{\circ}$, and $-175 \mathrm{~km} \mathrm{~s}^{-1}$ $<V_{\text {ss }}<-60 \mathrm{~km} \mathrm{~s}^{-1}$ (Wakker \& van Woerden 1991). The outer arm appears to lie between 15 and $20 \mathrm{kpc}$ from the Galactic center in this longitude range (Kulkarni, Blitz, \& Heiles 1982). At the latitudes of 3C 147 and 3C 380 the H I emission images from the LDSS show well-delineated structures in the longitude-velocity plane which can be associated with the outer arm. In addition, there are discrete $\mathrm{H} \mathrm{I}$ emission features in these images at the longitudes of 3C 147 and 3C 380. Corresponding to these features, the $\mathrm{H} \mathrm{I}$ emission spectra toward 3C 147 and 3C 380 from LDSS (Figs. $5 a$ and $6 a$ ) clearly show lines at $V_{1 \mathrm{sr}}=-120.4$ and -109.7 $\mathrm{km} \mathrm{s}{ }^{-1}$, respectively (Table 2). The high-velocity $\mathrm{H}_{\mathrm{I}}$ absorption lines detected in the current observations toward $3 \mathrm{C} 147\left(V_{\text {ss }}=-117.3\right.$ and $\left.-124.5 \mathrm{~km} \mathrm{~s}^{-1}\right)$ and $3 \mathrm{C}$ $380\left(V_{\text {lsr }}=-113.7 \mathrm{~km} \mathrm{~s}^{-1}\right)$ (Figs. $5 b$ and $\left.6 b\right)$ are associated with the respective $\mathrm{H}$ I emission features arising in the outer arm. As mentioned in $\S 3$, in both cases (i.e., 3C 147 and 3C 380 ) the high-velocity clouds have at least two temperature components - one with a spin temperature higher than or comparable to $1000 \mathrm{~K}$ and the second with a spin temperature lower than $200 \mathrm{~K}$. The higher temperature gas, presumably widespread, is observed in $\mathrm{H} \mathrm{I}$ emission. The narrow-line $\mathrm{H}$ I absorption may arise from smaller and colder regions ("clumps") embedded in this bigger structure. There are probably many such "clumps" in the highvelocity $\mathrm{H}$ I emission features since $\mathrm{H}_{\mathrm{I}}$ absorption was detected toward two out of the four extragalactic sources observed.

The heliocentric distances to these high-velocity $\mathrm{H}$ I clouds toward 3C 147 and 3C 380 are $10 \mathrm{kpc}$ and $18 \mathrm{kpc}$, respectively, assuming a mean galactocentric distance of 18 $\mathrm{kpc}$ to the outer arm (Kulkarni, Blitz, \& Heiles 1982; Blitz, Fich, \& Stark 1982). At these distances, the heights of these high-velocity clouds above the Galactic plane are 1.8 and 8 $\mathrm{kpc}$, respectively. Very little is known about the spin temperature of $\mathrm{H} \mathrm{I}_{\mathrm{I}}$ in the outer arm due to paucity of $\mathrm{H} \mathrm{I}$ absorption measurements. Payne, Salpeter, \& Terzian (1980) reported an $\mathrm{H}$ I absorption measurement in the outer arm toward $4 \mathrm{C} 33.48\left(l=66^{\circ} .4, b=8.4\right)$ at $V_{\mathrm{lsr}}=-128$ $\mathrm{km} \mathrm{s}^{-1}$ with $\tau=0.011$ and $T_{s}=69 \mathrm{~K}$. More recently, Akeson \& Blitz (1999) detected H I absorption in the outer arm toward the extragalactic sources $1901+319$ and $2005+403$. They report $\tau=0.012$ and 0.031 and $T_{s}=38$ and $147 \mathrm{~K}$ for these two lines of sight, respectively. The widths of these $\mathrm{H} \mathrm{I}$ absorption lines and the corresponding $\mathrm{H} \mathrm{I}$ emission lines are between 5 and $12 \mathrm{~km} \mathrm{~s}^{-1}$. Although the current $\mathrm{H}$ I absorption in the outer arm seems to imply the existence of similar spin temperature components, there are two distinct differences compared to the earlier two measurements: (1) the optical depths observed in the current observations are about a factor of 10 smaller than the previous measurements, and (2) the current measurements indicate the existence of multiple temperature com- 
ponents in the outer arm H I . The existence of multiple temperature components in other parts of the Galaxy has been well established based on earlier $\mathrm{H}$ I observations (Mebold et al. 1982; Stark et al. 1994). It appears that the less explored outer arm is not too different in this respect. However, the outer arm is different from the inner Galaxy in at least two aspects: (1) the column density of atomic hydrogen can be less than or equal to $10^{19} \mathrm{~cm}^{-2}$, and (2) the flux of diffuse Ly $\alpha$ radiation can be significantly lesser. These two factors play an important role in estimating the allowed range of spin temperatures for an allowed range of kinetic temperatures in the two-phase equilibrium models of Wolfire et al. (1995). It will be interesting to compare these estimates of spin temperatures with those obtained from observations toward many lines of sight in the outer arm.

\section{CONCLUSIONS}

We present observations of the Galactic $\mathrm{H}_{\text {I }} 21 \mathrm{~cm}$ line absorption toward four extragalactic sources using the WSRT. Toward one of the sources we have detected H I 21 $\mathrm{cm}$ line absorption in the warm neutral medium associated with the Perseus arm of the Galaxy. The observed optical depth is $(1.9 \pm 0.2) \times 10^{-3}$, leading to a spin temperature estimate of $3600 \pm 360 \mathrm{~K}$. Depending on the intensity of the diffuse $L y \alpha$ radiation field the kinetic temperature of the WNM is in the range $3600-7000 \mathrm{~K}$.

We have also detected $\mathrm{H}$ I $21 \mathrm{~cm}$ line absorption from high-velocity clouds in the outer arm of the Galaxy. We find multiple temperature components $\left(T_{s}<200 \mathrm{~K}\right.$ and $T_{s}>$ $1000 \mathrm{~K}$ ) in these high-velocity clouds similar to that observed in other parts of the Galaxy.

We thank the referee for a thorough reading of an earlier version of this paper and for giving detailed comments to improve the clarity and readability of the paper. The National Radio Astronomy Observatory (NRAO) is a facility of the National Science Foundation, operated under cooperative agreement by Associated Universities, Inc. The Westerbork Synthesis Radio Telescope is operated by the Netherlands Foundation for Research in Astronomy with financial support from the Netherlands Organization for Scientific Research (NWO). We thank Rene Vermeulen for scheduling and carrying out these observations.
Akeson, R. L., \& Blitz, L. 1999, ApJ, 523, 163

Blitz, L., Fich, M., \& Stark, A. A. 1982, ApJS, 49, 183

Carilli, C. L., Dwarakanath, K. S., \& Goss, W. M. 1998, ApJ, 502, L79

Clark, B. G. 1965, ApJ, 142, 1398

Clark, B. G., Radhakrishnan, V., \& Wilson, R. W. 1962, ApJ, 135, 151

Field, G. B. 1958, Proc. IRE, 46, 240

Hartmann, D., \& Burton, W. B. 1997, Atlas of Galactic Neutral Hydrogen (Cambridge: Cambridge Univ. Press)

Holberg, J. B. 1986, ApJ, 311, 969

Kalberla, P. M. W., Mebold, U., \& Reich, W. 1980, A\&A, 82, 275

Kulkarni, S. R., Blitz, L., \& Heiles, C. 1982, ApJ, 259, L63

Kulkarni, S. R., Heiles, C., Turner, K. C., \& Dickey, J. M. 1985, ApJS, 57, 631

Liszt, H. S. 2001, A\&A, 371, 698

Liszt, H. S., Dickey, J. M., \& Greisen, E. W. 1982, ApJ, 261, 102

\section{REFERENCES}

Mebold, U., \& Hills, D. 1975, A\&A, 42, 187

Mebold, U., Winnberg, A., Kalberla, P. M. W., \& Goss, W. M. 1981, A\&AS, 46, 389

. 1982, A\&A, 115, 223

Payne, H. E., Salpeter, E. E., \& Terzian, Y. 1980, ApJ, 240, 499 1983, ApJ, 272, 540

Radhakrishnan, V., Goss, W. M., Murray, J. D., \& Brooks, J. W. 1972, ApJS, 24, 49

Stark, R., Dickey, J. M., Burton, W. B., \& Wennmacker, A. 1994, A\&A, 281,199

Taylor, J. H., \& Cordes, J. M. 1993, ApJ, 411, 674

Vacca, W. D., Garmany, C. D., \& Shull, J. M. 1996, ApJ, 460, 914

Wakker, B. P., \& van Woerden, H. 1991, A\&A, 250, 509

Wolfire, M. G., Hollenbach, D., McKee, C. F., Tielens, A. G. G. M., \& Bakes, E. L. O. 1995, ApJ, 443, 152 